\title{
X-ray Hanbury Brown-Twiss interferometry for determination of ultrashort electron-bunch duration
}

\author{
Ichiro Inoue, ${ }^{1, *}$ Toru Hara, ${ }^{1}$ Yuichi Inubushi, ${ }^{1,2}$ Kensuke Tono, ${ }^{1,2}$ Takahiro Inagaki, ${ }^{1}$ \\ Tetsuo Katayama, ${ }^{1,2}$ Yoshiyuki Amemiya, ${ }^{3}$ Hitoshi Tanaka, ${ }^{1}$ and Makina Yabashi ${ }^{1,2}$ \\ ${ }^{1}$ RIKEN SPring-8 Center, 1-1-1 Kouto, Sayo, Hyogo 679-5148, Japan \\ ${ }^{2}$ Japan Synchrotron Radiation Research Institute, 1-1-1 Kouto, Sayo, Hyogo 679-5198, Japan \\ ${ }^{3}$ Department of Advanced Materials Science, Graduate School of Frontier Sciences, \\ The University of Tokyo, 5-1-5 Kashiwanoha, Kashiwa, Chiba 277-8561, Japan
}

(Received 2 September 2016; published 30 August 2018)

\begin{abstract}
An x-ray Hanbury Brown-Twiss interferometry to diagnose a temporal profile of a femtosecond electron bunch (e-bunch) is presented. We show that intensity interference of spontaneous x-ray radiation from the e-bunch reflects the e-bunch profile. Based on this relationship, a temporal profile of the 8.1-GeV e-bunch at SPring-8 Angstrom Compact free-electron LAser (SACLA) that generates X-ray free-electron laser (XFEL) light is characterized through the intensity interference measurement. Combining this e-bunch profile with a numerical simulation, the XFEL pulse duration generated by the e-bunch is estimated to be less than $10 \mathrm{fs}$.
\end{abstract}

DOI: 10.1103/PhysRevAccelBeams.21.080704

\section{INTRODUCTION}

Optical pulses and particle beams with ultrashort temporal duration, such as femtosecond electron pulses from a photocathode electron gun, attosecond laser light from high harmonic generation [1], and femtosecond synchrotron radiation produced by a laser-slicing technique [2], have been powerful probes for capturing ultrafast processes, which have provided new insights in various fields ranging in materials science, chemistry, and biology. An x-ray freeelectron laser (XFEL) based on the self-amplified spontaneous emission (SASE) scheme [3] is a newly developed ultrafast light source with femtosecond duration. The ultrafast nature of XFELs, in combination with their nearly full transverse coherence $[4,5]$ and high brilliance properties, has opened up innovative scientific opportunities, such as $\mathrm{x}$-ray structural determination based on a "diffraction-beforedestruction" scheme [6,7], investigation of nonlinear x-ray phenomena [8-11], and measurements and applications of femtosecond x-ray interactions with materials [12-15].

The ultrafast XFEL pulses are generated from the electron bunches (e-bunches) that are accelerated to a relativistic speed with strong compression to a femtosecond regime. Evaluation of the temporal profile of the e-bunch is therefore essential for optimizing accelerator parameters for

\footnotetext{
*inoue@spring8.or.jp
}

Published by the American Physical Society under the terms of the Creative Commons Attribution 4.0 International license. Further distribution of this work must maintain attribution to the author(s) and the published article's title, journal citation, and DOI. achieving XFEL amplification, as well as for characterizing the XFEL pulse duration, which is an important parameter for designing and analyzing experiments. In XFEL facilities, such as the Linac Coherent Light Source (LCLS) [16] and SPring-8 Angstrom Compact free-electron LAser (SACLA) [17], several techniques for temporal diagnostic of the e-bunch have been developed [18-23]. Among them, a radiofrequency transverse deflector ( $\mathrm{rf}$ deflector) is widely used due to its high temporal resolution and simplicity of the measurement [21-23]. In the rf deflector, the traveling, time-varying transverse rf field streaks the e-bunch and the temporal structure of the e-bunch is measured at a femtosecond time resolution.

At present, XFEL light sources are capable of generating sub-10 fs XFEL pulses [21,24]. In such cases, the e-bunch duration is comparable to or shorter than the time resolution of the e-bunch diagnostic schemes that are presently available. Furthermore, the concepts for generating attosecond XFELs are now rapidly emerging [25-28]. A new scheme to diagnose the e-bunch profile with an attosecond resolution is highly demanded.

In this paper, we present an X-ray intensity interferometry as a new temporal diagnostic scheme for e-bunches. Intensity interferometry, so called Hanbury-Brown Twiss (HBT) interferometry [29,30], is a technique to investigate statistical and coherence properties of light from the measurement of the intensity correlations. The HBT interferometry was extended to synchrotron $\mathrm{x}$-ray radiation 20 years ago [31,32], and the transverse coherence properties and the $\mathrm{x}$-ray pulse duration were evaluated with this technique [33-35]. More recently, the HBT interferometry of soft and hard XFELs was performed to characterize their statistical properties [36,37]. 
In the proposed scheme, spatial profiles of the spontaneous $\mathrm{x}$-ray beam generated by the e-bunch are measured in a shot-by-shot manner while changing the bandwidths using x-ray monochromators, and evaluate the degree of intensity interference via second-order intensity correlation functions. As is shown below, the degree of the interference is dominated by the magnitude relationship between the coherence time of the monochromatized x-ray beam and the e-bunch duration. Since the coherence time can be easily changed from picosecond to attosecond by using $\mathrm{x}$-ray monochromators, the x-ray HBT interferometry enables us to evaluate temporal profiles of the femtosecond or even attosecond e-bunches.

\section{PRINCIPLE}

The complex electric field of monochromatized spontaneous radiation from an e-bunch with a mean angular frequency $\bar{\omega}$ at a position $\boldsymbol{r}$ may be expressed as [38]

$$
E_{m}(\boldsymbol{r}, t)=F(\boldsymbol{r}, t) A(\boldsymbol{r}, t) \exp (i \bar{\omega} t),
$$

where $F(\boldsymbol{r}, t)$ is the field pulse envelop function, and $A(\boldsymbol{r}, t)$ is the Gaussian random variable. We assume $\left\langle A^{*}\left(\boldsymbol{r}_{1}, t\right) A\left(\boldsymbol{r}_{2}, t+\tau\right)\right\rangle$ can be factorized into functions of space $\mu\left(\boldsymbol{r}_{1}, \boldsymbol{r}_{2}\right)$ and time $\gamma_{t}(\tau)$. Here the brackets denote the ensemble average, which is experimentally replaced by average over different pulses. We note that this assumption is generally valid for monochromatized undulator radiations [39].

By introducing the Fourier component of the electric field of the x-ray beam before monochromator $\tilde{E}(\boldsymbol{r}, \omega)=$ $\int E(\boldsymbol{r}, t) e^{-i \omega t} d t$, the Fourier component of that after the monochromator can be expressed as $\tilde{E}_{m}(\boldsymbol{r}, \omega)=$ $R(\omega) \tilde{E}(\boldsymbol{r}, \omega)$ with the complex reflectivity of the monochromator $R(\omega)$.

In the x-ray HBT interferometry, we measure the time-integrated intensity for each X-ray pulse $I_{m}^{t}(\boldsymbol{r})=$ $\frac{1}{2} c \epsilon_{0} \int\left|E_{m}(\boldsymbol{r}, t)\right|^{2} d t$ with the speed of light $c$ and the permittivity of vacuum $\epsilon_{0}$. Then, the intensity correlation between different positions is evaluated with the normalized second-order intensity correlation function

$$
g^{(2)}\left(\boldsymbol{r}_{\mathbf{1}}, \boldsymbol{r}_{\mathbf{2}}\right)=\frac{\left\langle I_{m}^{t}\left(\boldsymbol{r}_{\mathbf{1}}\right) I_{m}^{t}\left(\boldsymbol{r}_{\mathbf{2}}\right)\right\rangle}{\left\langle\boldsymbol{I}_{m}^{t}\left(\boldsymbol{r}_{\mathbf{1}}\right)\right\rangle\left\langle I_{m}^{t}\left(\boldsymbol{r}_{\mathbf{2}}\right)\right\rangle} .
$$

We suppose that the normalized intensity envelope function $P(\boldsymbol{r}, t)=|F(\boldsymbol{r}, t)|^{2} / \int|F(\boldsymbol{r}, t)|^{2} d t$ is independent on $\boldsymbol{r}$ and is given by a summation of $N$ Gaussian functions $P(\boldsymbol{r}, t)=\sum_{n=1}^{N} p_{n} /\left(\sqrt{2 \pi} \sigma_{n}\right) \exp \left[-\left(t-t_{n}\right)^{2} /\left(2 \sigma_{n}^{2}\right)\right]$. In addition, we assume that the intensity reflectivity of monochromator $|R(\omega)|^{2}$ is given by $R_{0}^{2} \exp \left[-(\omega-\bar{\omega})^{2} /\left(2 \sigma_{\omega}^{2}\right)\right]$. Then, we obtain the relationship among $g^{(2)}$ between two separated positions $\left(\boldsymbol{r}_{1}, \boldsymbol{r}_{2}\right)$, the pulse envelope function, and the coherence time of the x-ray beam after monochromator $\tau_{c}=1 /\left(2 \sigma_{\omega}\right)$ as follows (see Supplemental Material [40]):

$$
\begin{aligned}
g^{(2)}\left(\boldsymbol{r}_{\mathbf{1}}, \boldsymbol{r}_{\mathbf{2}}\right)= & +\left|\mu\left(\boldsymbol{r}_{\mathbf{1}}, \boldsymbol{r}_{\mathbf{2}}\right)\right|^{2} \sum_{n=1}^{N} \sum_{m=1}^{N} \frac{p_{n} p_{m}}{\sqrt{1+\frac{\sigma_{n}^{2}+\sigma_{m}^{2}}{2 \tau_{c}^{2}}}} \\
& \times \exp \left[-\frac{1}{2} \cdot \frac{\left(t_{n}-t_{m}\right)^{2}}{\sigma_{n}^{2}+\sigma_{m}^{2}+2 \tau_{c}^{2}}\right] .
\end{aligned}
$$

In the case of $\boldsymbol{r}_{1}=\boldsymbol{r}_{2} \equiv \boldsymbol{r}$, the right side of Eq. (3) contains an additional term originating from photon shot noise $1 /\left\langle I_{\mathrm{ph}}(\boldsymbol{r})\right\rangle$, where $\left\langle I_{\mathrm{ph}}(\boldsymbol{r})\right\rangle$ is the average number of detected photons per pulse at $\boldsymbol{r}[35,41]$.

As seen from Eq. (3), the degree of the intensity interference is dominated by the magnitude relationship between $\sigma_{n}(n=1,2,3 \ldots N)$ and $\tau_{c}$. Thus, by measuring $g^{(2)}$ for different conditions where $\tau_{c}$ and $\sigma_{n}$ are comparable, one can readily evaluate the intensity envelop function of the x-ray pulse. Since the temporal electron density distribution, i.e., the current profile of the e-bunch $L(t)$, is related to $P(\boldsymbol{r}, t)$ via $P(\boldsymbol{r}, t)=L(t) / \int L(t) d t$, the current profile of the e-bunch can be determined when we know a total charge of the e-bunch $Q=\int L(t) d t$.

\section{EXPERIMENT}

Based on the above principle, we evaluated the current profile of the e-bunch with an energy of $8.1 \mathrm{GeV}$ that generated a $10.5 \mathrm{keV}$-XFEL pulse at SACLA BL3 [42]. The schematic illustration is shown in Fig. 1(a). Twentyone undulator segments are working for the normal operating condition to generate XFEL pulses, while only

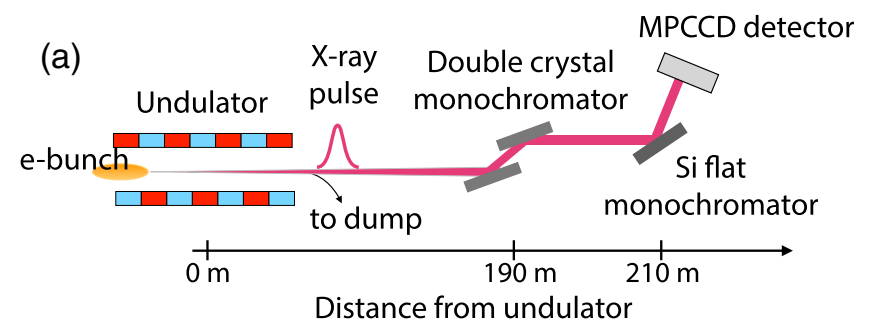

(b)
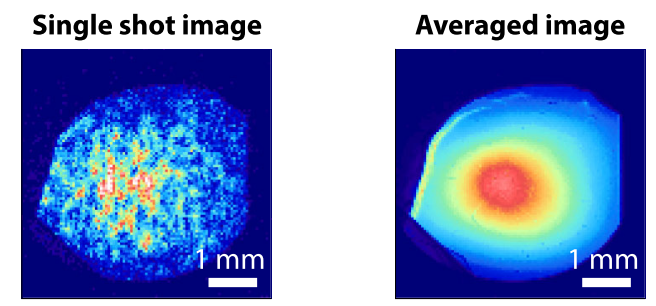

FIG. 1. (a) Schematic illustration of the x-ray HBT interferometry performed at SACLA BL3. (b) Single shot and averaged images of the monochromatized spontaneous radiation for the case of the flat Si crystal in a reflective index of 111. The edge of the $x$-ray beam profile originated from the limited apertures of the beamline optics. 
the most upstream undulator was employed for producing spontaneous radiation in this experiment by opening the magnetic gaps of the rest of the undulators. A silicon (Si) 111 double crystal monochromator in the optical hutch selected $10.5 \mathrm{keV}$ radiation with a bandwidth of $1 \mathrm{eV}$. The bandwidth was further reduced by another monochromator with a flat $\mathrm{Si}$ crystal in a reflective index of $111,220,311,440,333,444,660$, or 555 . For each experimental condition, the coherence time was determined by fitting the intensity reflectivity of monochromators $|R(\omega)|^{2}$ by a Gaussian function. The Gaussian function well describes the frequency dependence of the intensity reflectivity, which assures the validation of the determination of the electron bunch profile at the accuracy of the current experiment. The intensity profiles of the $\mathrm{x}$-ray beam after monochromator were measured in a pulse-by-pulse manner with a multiport charge coupled device detector [43].

\section{RESULTS AND DISCUSSION}

Figure 1(b) shows a typical single shot image and the averaged image over multiple pulses of the x-ray beam monochromatized by the $\mathrm{Si}$ flat crystals in a reflective index of 111 . The single shot image shows a grainy pattern, indicating the presence of the x-ray intensity interference between neighboring positions. Note that the grainy image is not due to the speckles caused by imperfections of the optical devices since the averaged image in Fig. 1(b) shows a smooth pattern.

We calculated $g^{(2)}$ between the central position of the reflected beam and the neighboring positions given by

$g^{(2)}(\Delta x, \Delta y)=\frac{\left\langle I_{m}^{t}\left(x_{0}+\Delta x, y_{0}+\Delta y\right) I_{m}^{t}\left(x_{0}, y_{0}\right)\right\rangle}{\left\langle I_{m}^{t}\left(x_{0}+\Delta x, y_{0}+\Delta y\right) I_{m}^{t}\left(x_{0}, y_{0}\right)\right\rangle}$,

where $x_{0}$ and $y_{0}$ are the central positions of the x-ray beam on the detector plane in the horizontal and vertical directions, respectively. Figure 2(a) shows $g^{(2)}$ measured with Si flat crystals in a reflective index of 111, 311, and 660. Apparently, $g^{(2)}$ increases for longer coherence times [ $\tau_{c}=0.8$ fs (Si 111), 2.6 fs (Si 311), and 30.9 fs (Si 660)]. Interestingly, $g^{(2)}$ decays faster in the horizontal direction than in the vertical direction, which is explained by the anisotropy of the electron beam emittance; the larger e-beam emittance in the horizontal direction compared with that in the vertical direction led a small transverse coherence length of the spontaneous radiation in the horizontal direction. The line profile of $g^{(2)}(\Delta y)=g^{(2)}(\Delta x=0 \mu \mathrm{m}, \Delta y)$ for each experimental configuration of the $\mathrm{Si}$ flat crystal was well described by a Gaussian function [Fig. 2(b)]. We determined the degree of the intensity interference $g_{0}^{(2)}$ by fitting $g^{(2)}(\Delta y)$ with a Gaussian function $g^{(2)}(\Delta y)=g_{0}^{(2)} \exp \left[-\frac{\Delta y^{2}}{2 l_{y}^{2}}\right]$. In the fitting process, the data point at $\Delta y=0 \mu \mathrm{m}$ was excluded to
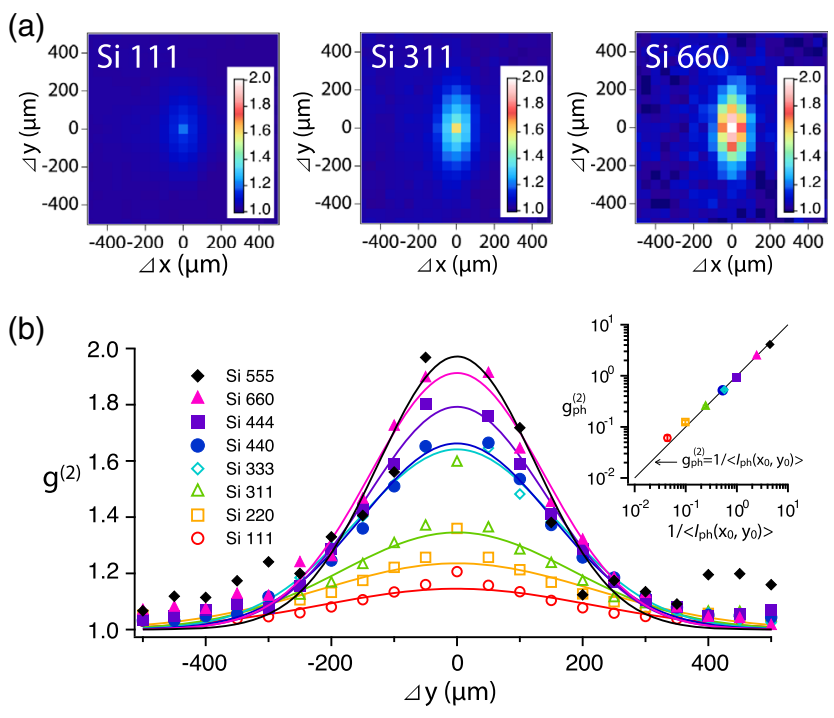

FIG. 2. (a) $g^{(2)}$ of the X-ray beam monochromatized by Si flat crystal in a reflective index of 111, 311, or 660. (b) Line profiles of $g^{(2)}(\Delta x=0 \mu \mathrm{m}, \Delta y)$ and their fitted results by Gaussian functions. Inset: degree of the effect of photon shot noise on intensity interference as a function of the reciprocal of average number of detected photons at the pixel corresponding to the center of the monochromatized $\mathrm{x}$-ray beam.

avoid the effect of photon shot noise on $g^{(2)}$. The degree of the effect of photon shot noise $g_{\mathrm{ph}}^{(2)}=g^{(2)}(\Delta x=0 \mu \mathrm{m}$, $\Delta y=0 \mu \mathrm{m})-g_{0}^{(2)}$ for each experimental configuration is plotted as a function of $1 /\left\langle I_{\mathrm{ph}}\left(x_{0}, y_{0}\right)\right\rangle$ in the inset of Fig. 2(b). As is predicted from theory, the values of $g_{\mathrm{ph}}^{(2)}$ are almost the same as $1 /\left\langle I_{\mathrm{ph}}\left(x_{0}, y_{0}\right)\right\rangle$.

To evaluate the e-bunch duration, we first assumed that the current profile is described by a single Gaussian function $\left(L(t)=\frac{Q}{\sqrt{2 \pi} \sigma} \exp \left[-\frac{t^{2}}{2 \sigma^{2}}\right]\right)$. Under such assumption, $g_{0}^{(2)}$ is given by

$$
g_{0}^{(2)}=1+\frac{1}{\sqrt{1+\sigma^{2} / \tau_{c}^{2}}} .
$$

We fit the measured $g_{0}^{(2)}$ as a function of the coherence time by Eq. (5) [Fig. 3(a)]. The fitting curve roughly describes the experimental result, and $\sigma$ was determined to be $8.8 \pm 0.8 \mathrm{fs}$, while we found a small discrepancy between the fitting curve and the experimental result. To improve the consistency between the experimental data and the model fitting, we considered the double Gaussian model of the current profile:

$L(t)=\frac{Q_{1}}{\sqrt{2 \pi} \sigma_{1}} \exp \left[-\frac{t^{2}}{2 \sigma_{1}^{2}}\right]+\frac{Q_{2}}{\sqrt{2 \pi} \sigma_{2}} \exp \left[-\frac{\left(t-t_{12}\right)^{2}}{2 \sigma_{2}^{2}}\right]$,

where $t_{12}$ is the time separation of the two Gaussian functions, and $Q_{1}\left(Q_{2}\right)$ and $\sigma_{1}\left(\sigma_{2}\right)$ are the total charges and the spread of the first (second) Gaussian function, 

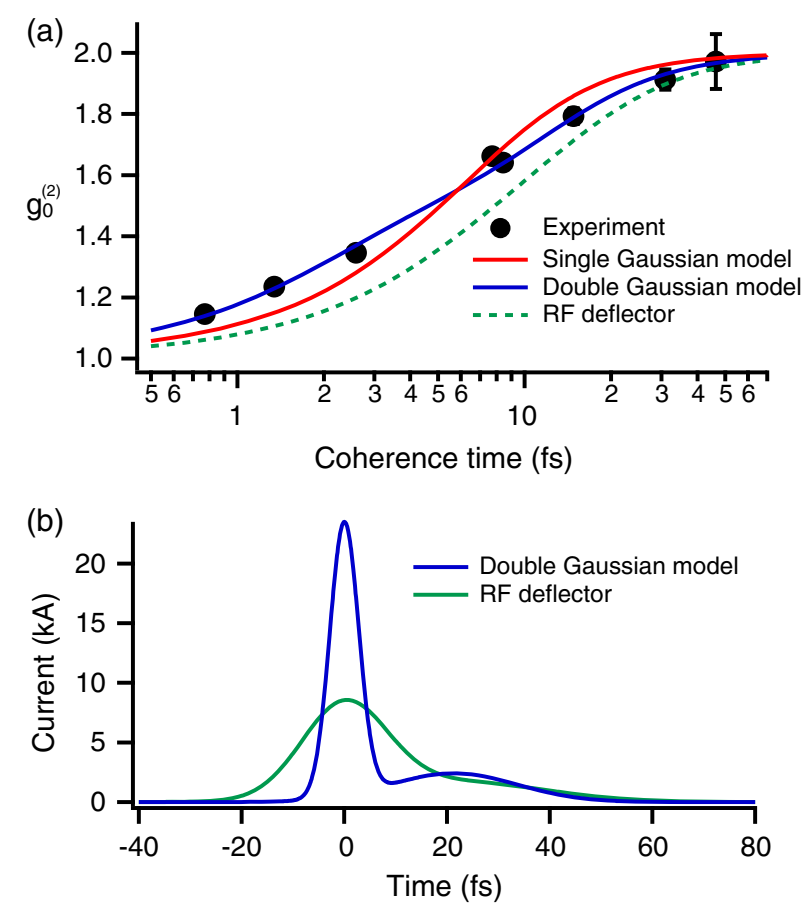

FIG. 3. (a) $g_{0}^{(2)}$ as a function of coherence time of the x-ray beam after monochromator (black markers) and the fitting results by single (red solid curve) and double (blue solid curve) Gaussian function models. Green dotted curve represents $g_{0}^{(2)}$ calculated by Eq. (7) with the e-bunch parameters measured by the rf deflector. (b) Current profile of the e-bunch determined by the x-ray HBT interferometry (blue solid curve) and that measured by the rf deflector (green solid curve).

respectively. Here, we set the time corresponding to the peak current position of the first Gaussian function to $0 \mathrm{fs}$ for simplicity. The current profile measured by the rf deflector at SACLA was well fitted by Eq. (6), and we obtained the following parameters: $Q_{1}=161 \pm 11 \mathrm{pC}$, $Q_{2}=75 \pm 7 \mathrm{pC}, \sigma_{1}=8.3 \pm 0.5 \mathrm{fs}, \sigma_{2}=17.3 \pm 1.1 \mathrm{fs}$, and $t_{12}=21.3 \pm 1.4$ fs. Here \pm denotes the pulse-by-pulse differences. In the double Gaussian model, the degree of the intensity interference is given by

$$
\begin{aligned}
g_{0}^{(2)}= & 1+\frac{Q_{1}^{2} /\left(Q_{1}+Q_{2}\right)^{2}}{\sqrt{1+\sigma_{1}^{2} / \tau_{c}^{2}}}+\frac{Q_{2}^{2} /\left(Q_{1}+Q_{2}\right)^{2}}{\sqrt{1+\sigma_{2}^{2} / \tau_{c}^{2}}} \\
& +\frac{2 Q_{1} Q_{2} /\left(Q_{1}+Q_{2}\right)^{2}}{\sqrt{1+\left(\sigma_{1}^{2}+\sigma_{2}^{2}\right) /\left(2 \tau_{c}^{2}\right)}} \exp \left[-\frac{1}{2} \cdot \frac{t_{12}^{2}}{\sigma_{1}^{2}+\sigma_{2}^{2}+2 \tau_{c}^{2}}\right] .
\end{aligned}
$$

We examined whether the double Gaussian model with parameters determined by the rf deflector is consistent with the result of the HBT interferometry. The green dotted curve in Fig. 3(a) is $g_{0}^{(2)}$ calculated by Eq. (7) with $Q_{1}=161 \mathrm{pC}, Q_{2}=75 \mathrm{pC}, \sigma_{1}=8.3 \mathrm{fs}, \sigma_{2}=17.3 \mathrm{fs}$, and $t_{12}=21.3 \mathrm{fs}$. While the calculated and the measured $g_{0}^{(2)}$ are almost the same for long coherence times over

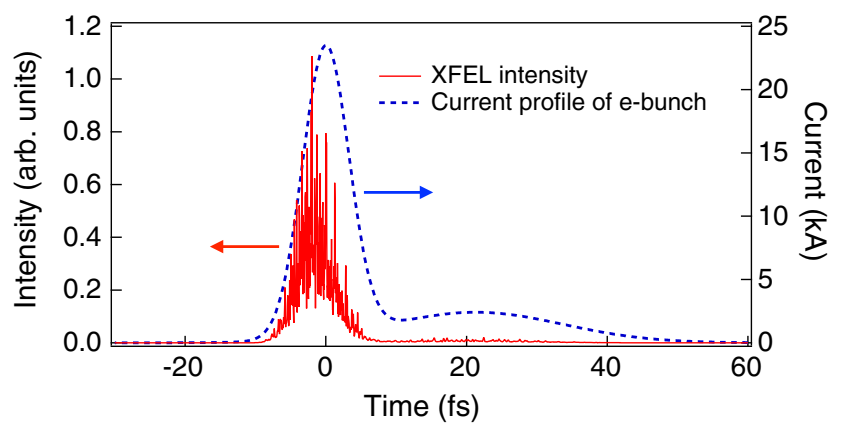

FIG. 4. Simulated temporal structure of the XFEL pulse (red solid curve) and the current profile of the e-bunch determined by the x-ray HBT interferometry (blue dotted curve).

$30 \mathrm{fs}$, the calculated value is much smaller than the measured $g_{0}^{(2)}$ for shorter coherence time. Such underestimation of $g_{0}^{(2)}$ could be explained by the presence of sharp current peak whose duration is shorter than the time resolution of the rf deflector.

Based on this assumption, we fitted the dependence of $g_{0}^{(2)}$ on the coherence time by Eq. (7) with two fitting paramets $\sigma_{1}$ and $\sigma_{2}$, while using the parameters measured by the rf deflector $Q_{1}=161 \mathrm{pC}, Q_{2}=75 \mathrm{pC}, t_{12}=$ $21.3 \mathrm{fs}$ that were not influenced by the limited temporal resolution of the rf deflector. The experimental result was well described by the fitting curve [Fig. 3(a)], and $\sigma_{1}$ and $\sigma_{2}$ were determined to be $2.8 \pm 0.3 \mathrm{fs}$ and $12.6 \pm 4.3 \mathrm{fs}$, respectively. The blue curve in Fig. 3(b) shows the current profile of the e-bunch determined by the x-ray $\mathrm{HBT}$ interferometry [Eq. (6) with $Q_{1}=161 \mathrm{pC}, Q_{2}=75 \mathrm{pC}$, $\sigma_{1}=2.8 \mathrm{fs}, \sigma_{2}=12.6 \mathrm{fs}$, and $t_{12}=21.3 \mathrm{fs}$. It was found that the peak current value of the e-bunch was $23 \mathrm{kA}$ and the peak duration was $7 \mathrm{fs}$ in full width of the halfmaximum (FWHM). For comparison, the current profile measured by the rf deflector is also shown in this figure.

Finally, we evaluated an emittance of the electron beam and the duration of the XFEL pulse generated by the e-bunch in the normal operating condition of SACLA with the help of a numerical simulation. Assuming the emittance is constant over all e-bunch positions and the current profile of the double Gaussian function model [Eq. (6) with $Q_{1}=161 \mathrm{pC}, Q_{2}=75 \mathrm{pC}, \sigma_{1}=2.8 \mathrm{fs}, \sigma_{2}=12.6 \mathrm{fs}$, and $t_{12}=21.3 \mathrm{fs}$, we simulated XFEL intensity evolution along the undulators in a normal operation condition at SACLA using a FEL simulation code SIMPLEX [44]. By performing simulation with different emittances, we found that the result with an emittance $\epsilon=0.9 \pm 0.1 \pi \mathrm{mm} \mathrm{mrad}$ well reproduces the intensity gain length $L_{g}=2.3 \mathrm{~m}$ that was obtained experimentally. Figure 4 shows the temporal profile of the XFEL pulse simulated at the exit of undulators for $\epsilon=0.9 \pi \mathrm{mm} \mathrm{mrad}$. For reference, the current profile of the e-bunch determined by the HBT interferometry is also shown. It was found that the high current region of the e-bunch beneath $0 \mathrm{fs}$ in the horizontal 
axis emitted intense XFEL radiation, while the contribution of the rest part of the e-bunch to the XFEL intensity was negligibly small. Accordingly, the XFEL pulse duration was evaluated to be 6.3 fs (FWHM), which is consistent with the previous evaluation on the XFEL pulse duration at SACLA $[9,24]$.

\section{SUMMARY}

In this paper, an x-ray HBT interferometry to diagnose temporal profiles of e-bunches is proposed. The e-bunch profile at SACLA was determined by using the scheme, and the duration of the XFEL pulse generated by the e-bunch was estimated by combining the determined e-bunch profile with a numerical simulation.

The x-ray HBT interferometry is based on a simple and robust principle, and is easily conducted without a complex experimental setup. This method should be highly effective for precise e-bunch diagnostics and contributes to controlling time structure of the e-bunch for achieving XFEL amplification. Furthermore, the x-ray HBT interferometry is applicable to much shorter e-bunches, because the coherence time of the x-ray beam can be reduced down to a single attosecond region using multilayer optics [45] that are widely used as an optical component at synchrotron $\mathrm{x}$-ray facilities [46-48]. The method would readily provide a pathway to evaluate relativistic attosecond electron bunches, which is beneficial not only for XFEL science but for advancing technology of plasma wakefield electron acceleration [49].

\section{ACKNOWLEDGMENTS}

We acknowledge Dr T. Tanaka and Dr. K. Tamasaku (RIKEN) for valuable advice and extensive discussions. This study was partially supported by JSPS KAKENHI Grant No. 17K14137. One of the authors (I. I.) acknowledges support from SACLA graduate-course student program.

[1] T. Brabec and F. Krausz, Intense few-cycle laser fields: Frontiers of nonlinear optics, Rev. Mod. Phys. 72, 545 (2000).

[2] R. W. Schoenlein et al., Generation of femtosecond pulses of synchrotron radiation, Science 287, 2237 (2000).

[3] B. W. McNeil and N. R. Thompson, X-ray free-electron lasers, Nat. Photonics 4, 814 (2010).

[4] C. Gutt et al., Single Shot Spatial and Temporal Coherence Properties of the SLAC Linac Coherent Light Source in the Hard X-Ray Regime, Phys. Rev. Lett. 108, 024801 (2012).

[5] I. Inoue et al., Characterizing transverse coherence of an ultra-intense focused X-ray free-electron laser by an extended Young's experiment, IUCrJ 2, 620 (2015).

[6] R. Neutze, R. Wouts, D. van der Spoel, E. Weckert, and J. Hajdu, Potential for biomolecular imaging with femtosecond X-ray pulses, Nature (London) 406, 752 (2000).
[7] H. N. Chapman et al., Femtosecond X-ray protein nanocrystallography, Nature (London) 470, 73 (2011).

[8] T. E. Glover et al., X-ray and optical wave mixing, Nature (London) 488, 603 (2012).

[9] K. Tamasaku et al., Double Core-Hole Creation by Sequential Attosecond Photoionization, Phys. Rev. Lett. 111, 043001 (2013).

[10] K. Tamasaku et al., X-ray two-photon absorption competing against single and sequential multiphoton processes, Nat. Photonics 8, 313 (2014).

[11] S. Shwartz et al., X-Ray Second Harmonic Generation, Phys. Rev. Lett. 112, 163901 (2014).

[12] H. Yoneda, Y. Inubushi, M. Yabashi, T. Katayama, T. Ishikawa, H. Ohashi, H. Yumoto, K. Yamauchi, H. Mimura, and H. Kitamura, Saturable absorption of intense hard X-rays in iron, Nat. Commun. 5, 5080 (2014).

[13] H. Yoneda et al., Atomic inner-shell laser at 1.5-ångström wavelength pumped by an X-ray free-electron laser, Nature (London) 524, 446 (2015).

[14] I. Inoue et al., Observation of femtosecond X-ray interactions with matter using an X-ray-X-ray pump-probe scheme, Proc. Natl. Acad. Sci. U.S.A. 113, 1492 (2016).

[15] K. Ferguson et al., Transient lattice contraction in the solidto-plasma transition, Sci. Avd. 2, 1500837 (2016).

[16] P. Emma et al., First lasing and operation of an ångstromwavelength free-electron laser, Nat. Photonics 4, 641 (2010).

[17] T. Ishikawa et al., A compact X-ray free-electron laser emitting in the sub-ångström region, Nat. Photonics 6, 540 (2012).

[18] Y. Ding, C. Behrens, P. Emma, J. Frisch, Z. Huang, H. Loos, P. Krejcik, and M.-H. Wang, Femtosecond X-ray pulse temporal characterization in free-electron lasers using a transverse deflector, Phys. Rev. ST Accel. Beams 14, 120701 (2011).

[19] Z. Huang et al., in Proceedings of the 24th Particle Accelerator Conference, PAC-2011, New York, 2011 (IEEE, New York, 2011), p. 2459.

[20] T. J. Maxwell, C. Behrens, Y. Ding, A. S. Fisher, J. Frisch, Z. Huang, and H. Loos, Coherent-Radiation Spectroscopy of Few-Femtosecond Electron Bunches Using a MiddleInfrared Prism Spectrometer, Phys. Rev. Lett. 111, 184801 (2013).

[21] C. Behrens et al., Few-femtosecond time-resolved measurements of X-ray free-electron lasers, Nat. Commun. 5, 3762 (2014).

[22] Y. Otake, H. Maesaka, S. Matsubara, S. Inoue, K. Yanagida, H. Ego, C. Kondo, T. Sakurai, T. Matsumoto, and H. Tomizawa, Beam monitor system for an x-ray free electron laser and compact laser, Phys. Rev. ST Accel. Beams 16, 042802 (2013).

[23] H. Ego, H. Maesaka, T. Sakurai, Y. Otake, T. Hashirano, and S. Miura, Transverse C-band deflecting structure for longitudinal electron-bunch-diagnosis in XFEL "SACLA", Nucl. Instrum. Methods Phys. Res., Sect. A 795, 381 (2015).

[24] Y. Inubushi et al., Determination of the Pulse Duration of an X-Ray Free Electron Laser Using Highly Resolved Single-Shot Spectra, Phys. Rev. Lett. 109, 144801 (2012).

[25] N. R. Thompson and B. W. J. McNeil, Mode Locking in a Free-Electron Laser Amplifier, Phys. Rev. Lett. 100, 203901 (2008). 
[26] T. Tanaka, Proposal for a Pulse-Compression Scheme in X-Ray Free-Electron Lasers to Generate a Multiterawatt, Attosecond X-Ray Pulse, Phys. Rev. Lett. 110, 084801 (2013).

[27] D. J. Dunning, B. W. J. McNeil, and N. R. Thompson, Proposal for a Pulse-Compression Scheme in X-Ray FreeElectron Lasers to Generate a Multiterawatt, Attosecond X-Ray Pulse, Phys. Rev. Lett. 110, 104801 (2013).

[28] R. Prat and S. Reiche, Simple Method to Generate Terawatt-Attosecond X-Ray Free-Electron-Laser Pulses, Phys. Rev. Lett. 114, 244801 (2015).

[29] R. Hanbury Brown and R. Q. Twiss, Correlation between photons in two coherent beams of light, Nature (London) 177, 27 (1956).

[30] R. Hanbury Brown and R. Q. Twiss, A test of a new type of stellar interferometer on sirius, Nature (London) 178, 1046 (1956).

[31] Y. Kunimune, Y. Yoda, K. Izumi, M. Yabashi, X.-W. Zhang, T. Harami, M. Ando, and S. Kikuta, Two-photon correlations in $\mathrm{X}$-rays from a synchrotron radiation source, J. Synchrotron Radiat. 4, 199 (1997).

[32] E. Gluskin, E. E. Alp, I. McNulty, W. Sturhahn, and J. Sutter, A classical Hanbury Brown-Twiss experiment with hard X-rays, J. Synchrotron Radiat. 6, 1065 (1999).

[33] M. Yabashi, K. Tamasaku, and T. Ishikawa, Characterization of the Transverse Coherence of Hard Synchrotron Radiation by Intensity Interferometry, Phys. Rev. Lett. 87, 140801 (2001).

[34] M. Yabashi, K. Tamasaku, and T. Ishikawa, Measurement of X-Ray Pulse Widths by Intensity Interferometry, Phys. Rev. Lett. 88, 244801 (2002).

[35] A. Singer et al., Intensity Interferometry of Single X-Ray Pulses from a Synchrotron Storage Ring, Phys. Rev. Lett. 113, 064801 (2014).

[36] A. Singer et al., Hanbury Brown-Twiss Interferometry at a Free-Electron Laser, Phys. Rev. Lett. 111, 034802 (2013).

[37] S. Song et al., Intensity interferometry measurements with hard x-ray FEL pulses at the Linac Coherent Light Source, Proc. SPIE Int. Soc. Opt. Eng. 9210, 10 (2014).
[38] Y. Miyamoto, T. Kuga, M. Baba, and M. Matsuoka, Measurement of ultrafast optical pulses with two-photon interference, Opt. Lett. 18, 900 (1993).

[39] G. Geloni, E. Saldin, E. Schneidmiller, and M. Yurkov, Transverse coherence properties of X-ray beams in thirdgeneration synchrotron radiation sources, Nucl. Instrum. Methods Phys. Res., Sect. A 588, 463 (2008).

[40] See Supplemental Material at http://link.aps.org/ supplemental/10.1103/PhysRevAccelBeams.21.080704 for derivation of the relationship between coherence time and degree of intensity interference.

[41] L. Mandel and E. Wolf, Optical Coherence and Quantum Optics (Cambridge University Press, Cambridge, England, 1995).

[42] K. Tono et al., Beamline, experimental stations and photon beam diagnostics for the hard $\mathrm{x}$-ray free electron laser of SACLA, New J. Phys. 15, 083035 (2013).

[43] T. Kameshima et al., Development of an X-ray pixel detector with multi-port charge-coupled device for X-ray free-electron laser experiments, Rev. Sci. Instrum. 85, 033110 (2014).

[44] T. Tanaka, Proc. FEL 2004, 435 (2004).

[45] D. Attwood, Soft X-rays and Extreme Ultraviolet Radiation: Principles and Applications (Cambridge University Press, Cambridge, England, 2007).

[46] Y.S. Chu, Performance of a double-multilayer monochromator at Beamline 2-BM at the Advanced Photon Source, Rev. Sci. Instrum. 73, 1485 (2002).

[47] M. Stampanoni, A. Groso, A. Isenegger, G. Mikuljan, Q. Chen, D. Meister, M. Lange, R. Betemps, S. Henein, and R. Abela, TOMCAT: A beamline for TOmographic Microscopy and Coherent rAdiology experimenTs, AIP Conf. Proc. 879, 848 (2007).

[48] A. Rack et al., Comparative study of multilayers used in monochromators for synchrotron-based coherent hard X-ray imaging, J. Synchrotron Radiat. 17, 496 (2010).

[49] E. Esarey, C. B. Schoroeder, and W. P. Leemans, Physics of laser-driven plasma-based electron accelerators, Rev. Mod. Phys. 81, 1229 (2009). 